\title{
NEW DISTRIBUTED POWER CONTROL ALGORITHMS FOR MOBILE COMMUNICATIONS
}

\author{
R. A. de Oliveira Neto, F. de S. Chaves, F. R. P. Cavalcanti, T. F. Maciel
}

\begin{abstract}
Most distributed power control algorithms have been proposed assuming constant interference and constant path gain. These considerations may result in lower performance gains in fast time-varying channel conditions. In this work, we present two algorithms that address this problem efficiently outperforming the classical Distributed Power Control (DPC) algorithm.
\end{abstract}

Keywords: power control, fading channels, cellular radio.

Resumo - A maioria dos algoritmos de controle distribuído de potência têm sido propostos assumindo o ganho de percurso e a interferência constantes. Estas considerações podem resultar em baixos ganhos de desempenho em condições de canal variante no tempo. Neste trabalho, apresentamos dois algoritmos que tratam deste problema eficientemente e superam o algoritmo clássico DPC.

Palavras-chave: controle de potência, canais com desvanecimento, rádio celular.

\section{INTRODUCTION}

In cellular wireless systems, good communication can be efficiently provided by ensuring just a minimum signal quality for individual connections. By appropriately adjusting transmission power levels, minimum link quality requirements can be attained without incurring in unnecessary interference generation. This technique is called power control.

However. the employment of this technique is not trivial when we strive with a multipath environment, where fast fading occurs, since SINR (Signal-to-Interference plus Noise Ratio) depends on the path gain and the co-channel interference, which are influenced by fast fading. Fast fading, also called short-term fading or multipath fading, is the phenomenon that describes the rapid amplitude fluctuations of a radio signal over a short period of time or travel distance. These rapid fluctuations cause degradation in the action of power control [1].

Some papers have studied the performance of power control in fast fading environment. In [2], a new algorithm is derived from the classical Distributed Power Control (DPC) algorithm [3], considering a time-varying path gain. In [4], a neural network is used to predict the future channel

R. A. de Oliveira Neto, F. de S. Chaves, F. R. P. Cavalcanti and T. F. Maciel are with Grupo de Pesquisa em Telecomunicações Sem-Fio (GTEL). Universidade Federal do Ceará (UFC). (E-mails: $\{$ neto, fabiano, rodrigo, maciel\}@gtel.ufc.br) conditions. Similarly, [5] and [6] use adaptive filters, with tap weights updated by least-mean-square (LMS) and recursive least-square (RLS) algorithms, respectively, in order to predict the future path gain.

In this work, two new distributed power control algorithms are presented, outperforming the classical DPC algorithm [3] and Lee algorithm [2] when a time-varying channel is considered. These new algorithms differ from the DPC in that its deduction assumes both path gain and interference to be time-varying functions.

\section{DPC ALGORITHM}

DPC algorithm can be deducted from a differential dynamic that makes the SINR of each user evolve towards a target SINR value by an amount proportional to the offset from its current SINR. This dynamic can be expressed by the following time-continuous differential equation [3]:

$$
\rho_{i}^{\prime}(t)=-\beta\left[\rho_{i}(t)-\rho_{i}\right]
$$

where the upper symbol ' in $\rho_{i}(t)$ denotes the derivative with respect to time, $\beta$ is a positive constant between 0 and 1 which controls the convergence rate of the algorithm, $\rho_{t}$ is the target SINR and $\rho_{i}(t)$ is the instantaneous SINR of the $i^{\text {th }}$ link expressed by:

$$
\rho_{i}(t)=\frac{g_{i}(t) \cdot p_{i}(t)}{I_{i}(t)}
$$

where $g_{1}(t)$ is the path gain, $p_{i}(t)$ is the transmitter power and $l_{j}(t)$ is the total interference (including noise). The total interference $I_{i}(t)$ is defined as:

$$
I_{i}(t)=\sum_{j \neq i} g_{j}(t) \cdot p_{j}(t)+\nu
$$

where $\nu$ is the thermic additive noise.

Considering only the SINR to be time-variant, that is, assuming that the path gain and interference do not vary between two consecutive iterations, the algorithm becomes:

$$
p_{i}(k+1)=p_{i}(k)\left[(1-\beta)+\beta \cdot \frac{\rho_{t}}{\rho_{i}(k)}\right] .
$$

In [3], it is proved that when $\beta=1$, the convergence speed is higher. So, setting $\beta=1$, eq. (4) becomes: 


$$
p_{i}(k+1)=p_{i}(k) \cdot \frac{\rho_{t}}{\rho_{i}(k)}=\rho_{t} \cdot \frac{I_{i}(k)}{g_{i}(k)} .
$$

When we use this algorithm in a time-varying channel, according to eq. (2), the SINR in instant $k+1$ is:

$$
\rho_{i}(k+1)=\rho_{t} \cdot \frac{g_{i}(k+1)}{g_{i}(k)} \cdot \frac{I_{i}(k)}{I_{i}(k+1)} .
$$

As seen in eq. (6), the result of DPC algorithm is disturbed by path gain and interference variations. In order to overcome this problem, we derive new algorithms that can be considered evolutions of the DPC algorithm.

\section{NEW DPC ALGORITHMS}

In this section, we propose two new distributed power control algorithms that can be considered improvements of the classical DPC algorithm [3].

\subsection{ALGORITHM 1}

In eq. (6), we can see that the precision of balancing the SINR of the $i^{\text {th }}$ link is disturbed by terms $\frac{g_{i}(k+1)}{g_{i}(k)}$ and $\frac{I_{1}(k)}{I_{2}(k+1)}$. In order to cancel these terms, we proposed a simple modification in eq. (5). If the rate of power control commands is higher than the channel fading rate, then we can use the following approximations:

$$
\left[\frac{g_{i}(k+1)}{g_{i}(k)}\right]^{-1} \approx \frac{g_{i}(k-1)}{g_{i}(k)}
$$

and,

$$
\left[\frac{I_{i}(k)}{I_{i}(k+1)}\right]^{-1} \approx \frac{I_{i}(k)}{I_{i}(k-1)}
$$

Based on these approximations, the algorithm 1 is given by:

$$
\begin{aligned}
p_{i}(k+1) & =\rho_{t} \cdot \frac{I_{i}(k)}{g_{i}(k)} \cdot \frac{g_{i}(k-1)}{g_{i}(k)} \cdot \frac{I_{i}(k)}{I_{i}(k-1)} \\
& =\rho_{t} \cdot \frac{g_{i}(k-1)}{g_{i}^{2}(k)} \cdot \frac{I_{i}^{2}(k)}{I_{i}(k-1)} .
\end{aligned}
$$

So, the obtained SINR in discrete time $k+1$ is:

$$
\begin{aligned}
& \rho_{i}(k+1)= \\
& \rho_{t} \cdot \frac{g_{i}(k-1) \cdot g_{i}(k+1)}{g_{i}^{2}(k)} \cdot \frac{I_{i}^{2}(k)}{I_{i}(k-1) \cdot I_{i}(k+1)} .
\end{aligned}
$$

Therefore, for an adequate power control command rate, according to the approximations in eqs. (7) and (8), we have $\rho_{i}(k+1) \approx \rho_{t}, \forall k$.

\subsection{ALGORITHM 2}

The discrete-time SINR $\rho_{i}(k)$ of a link is given by:

$$
\rho_{i}(k)=\frac{g_{i}(k) \cdot p_{i}(k)}{I_{i}(k)}
$$

So. the instantaneous transmit power necessary to balance this link for $\rho_{i}(k)=\rho_{t}$, for all instants $k$, satisfies the following equation:

$$
\rho_{t}=\frac{g_{i}(k) \cdot p_{i}(k)}{I_{i}(k)} \Rightarrow p_{i}(k)=\frac{\rho_{1} \cdot I_{i}(k)}{g_{i}(k)}
$$

We do not predispose of the values of $I_{i}(k)$ and $g_{i}(k)$, because these are instantaneous values. In order to solve this problem, we propose a simple prediction method based on Taylor's Series.

Taylor's Series is used to expand continuous functions $f(x)$ in the following form [7]:

$$
f(x)=f\left(x_{0}\right)+\sum_{n=1}^{\infty} \frac{f^{(n)}\left(x_{0}\right) \cdot\left(x-x_{0}\right)^{n}}{n !}
$$

where the term $f^{(n)}(x)$ represents the $n^{\text {th }}$ derivative of $f(x)$ with respect to $x$. Due to $\left(x-x_{0}\right)^{n}$ and $n !$, when $x$ and $x_{0}$ are adjacent values, the higher order terms can be neglected. Thus, keeping only the first two terms of the series, we have:

$$
f(x) \approx f\left(x_{0}\right)+f^{\prime}\left(x_{0}\right) \cdot\left(x-x_{0}\right) .
$$

Now, we transform (14) into a discrete time equation. For this, we assume that $x_{0}$ is the current discrete time instant $k$ and $x$ the next instant $k+1$. Further, $f^{\prime}\left(x_{0}\right)$ is substituted by $f(k)-f(k-1)$. In this way, we obtain:

$$
f(k+1) \approx 2 \cdot f(k)-f(k-1)
$$

Therefore, we can use (15) in order to predict the path gain and interference:

$$
\begin{gathered}
\hat{g}_{i}(k+1)=2 \cdot g_{i}(k)-g_{i}(k-1), \\
\hat{I}_{i}(k+1)=2 \cdot I_{i}(k)-I_{i}(k-1) .
\end{gathered}
$$

So, using (12), (16) and (17), the transmit power at instant $(k+1)$ is expressed by the following proposed algorithm:

$p_{i}(k+1)=\rho_{t} \cdot \frac{\widehat{I}_{i}(k+1)}{\widehat{g}_{i}(k+1)}=\rho_{t} \cdot\left[\frac{2 \cdot I_{i}(k)-I_{i}(k-1)}{2 \cdot g_{i}(k)-g_{i}(k-1)}\right]$.

The obtained SINR in discrete time $k+1$ is: 


$$
\rho_{i}(k+1)=\rho_{t} \cdot \frac{\hat{I}_{i}(k+1)}{I_{i}(k+1)} \cdot \frac{g_{i}(k+1)}{\hat{g}_{i}(k+1)} .
$$

Note that when the estimates tend to correct values, that is, $\hat{g}(k+1) \approx g(k+1)$ and $\hat{I}(k+1) \approx I(k+1)$, the SINR tends to $\rho_{t}$.

\section{RELATIONSHIP BETWEEN PROPOSED ALGORITHMS AND DPC}

It is interesting to compare the DPC algorithm with algorithms 1 and 2. Algorithm 1 (eq. 9) comes directly from eq. (5) using eqs. (7) and (8). The obtained SINR is expressed in (10).

Analyzing eq. (10), we can conclude that algorithm 1 works better when the two sequences $g_{i}(k-1), g_{i}(k)$, $g_{i}(k+1)$ and $I_{i}(k-1), I_{i}(k), I_{i}(k+1)$ vary similarly to a geometric progression. This can be seen in eqs. (7) and (8), where according to these approximations, the term of time $k$ is the geometric mean of terms of time $k-1$ and $k+1$. As in algorithm 2, it is possible to interpret eqs. (7) and (8) like predictions of the terms $g_{i}(k+1)$ and $I_{i}(k+1)$ :

$$
\begin{aligned}
& \widehat{g}_{i}(k+1)=\frac{g_{i}^{2}(k)}{g_{i}(k-1)}, \\
& \widehat{I}_{i}(k+1)=\frac{I_{i}^{2}(k)}{I_{i}(k-1)} .
\end{aligned}
$$

With respect to algorithm 2, it has the same format of DPC, in spite of the fact that DPC is deducted from a differential equation. The update equation of DPC can be written simply as:

$$
p_{i}(k+1)=\rho_{l} \cdot \frac{I_{i}(k)}{g_{i}(k)},
$$

while the proposed algorithm can be expressed as:

$$
p_{i}(k+1)=\frac{\rho_{t} \cdot \widehat{I}_{i}(k+1)}{\widehat{g}_{i}(k+1)} .
$$

The difference comes from the fact that the standard DPC algorithm is based on past information about path gain and interference, causing performance degradation.

The obtained SINR by algorithm 2 is expressed in eq. (19). Expanding this equation we have:

$$
\begin{aligned}
& \rho_{i}(k+1)= \\
& \rho_{t} \cdot \frac{2 \cdot I_{i}(k)-I_{i}(k-1)}{I_{i}(k+1)} \cdot \frac{g_{i}(k+1)}{2 \cdot g_{i}(k)-g_{i}(k-1)} .
\end{aligned}
$$

Analyzing the above equation, we can conclude that the algorithm 2 works better when the two sequences $g_{i}(k-1)$, 67 $g_{i}(k), g_{i}(k+1)$ and $I_{i}(k-1), I_{i}(k), I_{i}(k+1)$ vary similarly to an arithmetic progression. This can be seen in eqs. (16) and (17), where according to these approximations the term of time $k$ is the arithmetic mean of terms of time $k-1$ and $k+1$.

\section{SIMULATION RESULTS}

We now illustrate the performance of the proposed algorithms by simulations in the context of a single cell CDMA (Code Division Multiple Access) system in the uplink direction.

\subsection{SIMULATION MODEL}

A snapshot simulation model is assumed where mobile stations are uniformly distributed over the cell area. The actuation period of the power control algorithm is $1 \mathrm{~ms}$, unless otherwise stated. Other simulation parameters are set as follows. The cell radius is set to $1.5 \mathrm{~km}$ and omnidirectional antennas are considered. A simplified path loss model is used, where $P L(d)=120+$ $40 \log _{10}(d)[\mathrm{dB}]$. The distance $d$ is expressed in kilometers and represents the distance between mobile and base stations. Shadowing standard deviation is assumed $6 \mathrm{~dB}$. Fast fading is implemented following Jakes model [8] with three different Doppler spreads: $18.5 \mathrm{~Hz}, 55.5 \mathrm{~Hz}$ and $92.5 \mathrm{~Hz}$. It is assumed that the measurements $g_{i}(t)$ and $I_{i}(t)$ are exact for each algorithm.

The processing gain of the simulated CDMA system is assumed $21 \mathrm{~dB}$ and the noise power is set to $-110 \mathrm{dBm}$. The target SINR $\rho_{t}$ for all algorithms is set to $-12 \mathrm{~dB}$ resulting in a target bit energy per interference spectral density ratio, $\left(E_{b} / N_{0}\right)_{t}$, of $9 \mathrm{~dB}$ after despreading. Maximum mobile station transmit power is limited to $21 \mathrm{dBm}$ and the initial transmit power is set to the minimum transmit power $(-70$ $\mathrm{dBm})$.

\subsection{LINK-LEVEL RESULTS}

Fig. 1 shows a sample of the $E_{b} / N_{0}$ evolution achieved by a given user in a typical snapshot for DPC, Lee algorithm, algorithms 1 and 2 . In this case, ten mobile stations are placed in the cell and the same system configuration and fading realizations are used for all algorithms, with Doppler spread $55.5 \mathrm{~Hz}$.

From fig. 1, it is clearly observable that the proposed algorithms are able to stabilize the $E_{b} / N_{0}$ around the target $\left(E_{b} / N_{0}\right)_{t}$ better than DPC algorithm and Lee algorithm. In other words, the mean squared error (MSE) between the actual and the target $E_{b} / N_{0}$ is smaller for the proposed algorithms than for DPC and Lee algorithm. This behavior was observed for all snapshots and other values of Doppler spreads, as it is shown in table 1 , in which it is also presented the MSE for Doppler spreads $18.5 \mathrm{~Hz}$ and $92.5 \mathrm{~Hz}$, for the same configurations used in fig. 1 . 


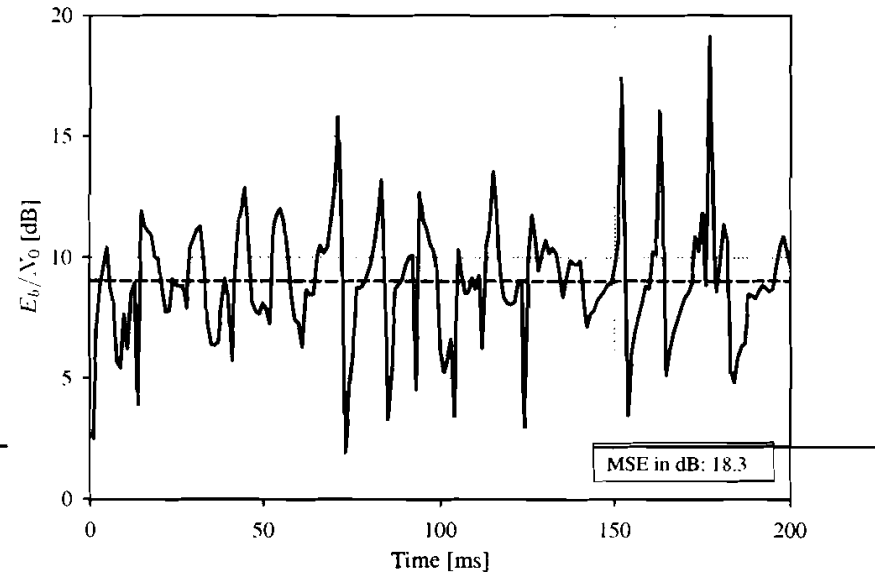

(a) DPC algorithm.

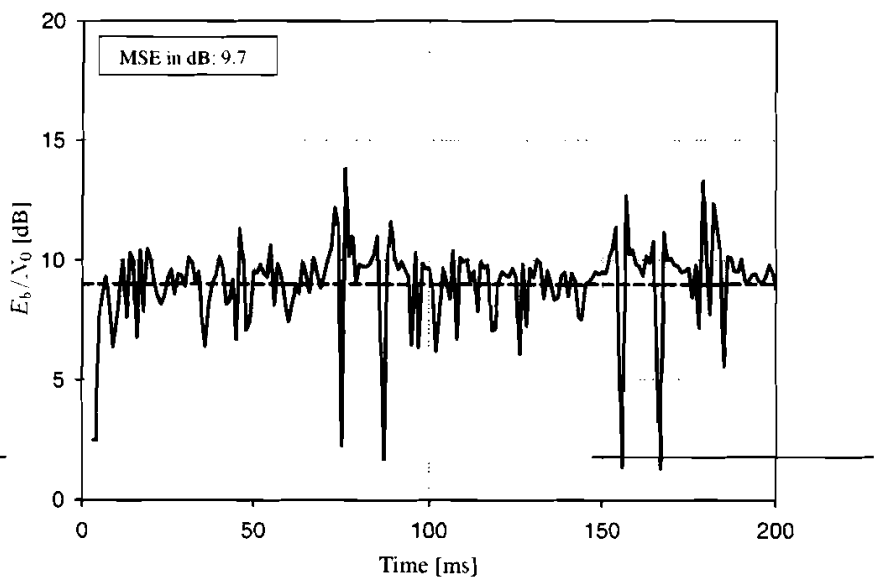

(c) Algorithm 1.

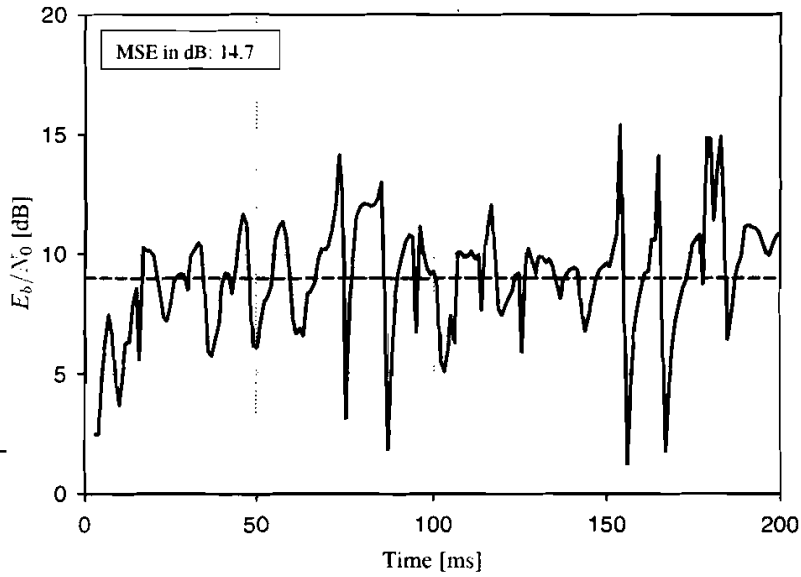

(b) Lee Algorithm.

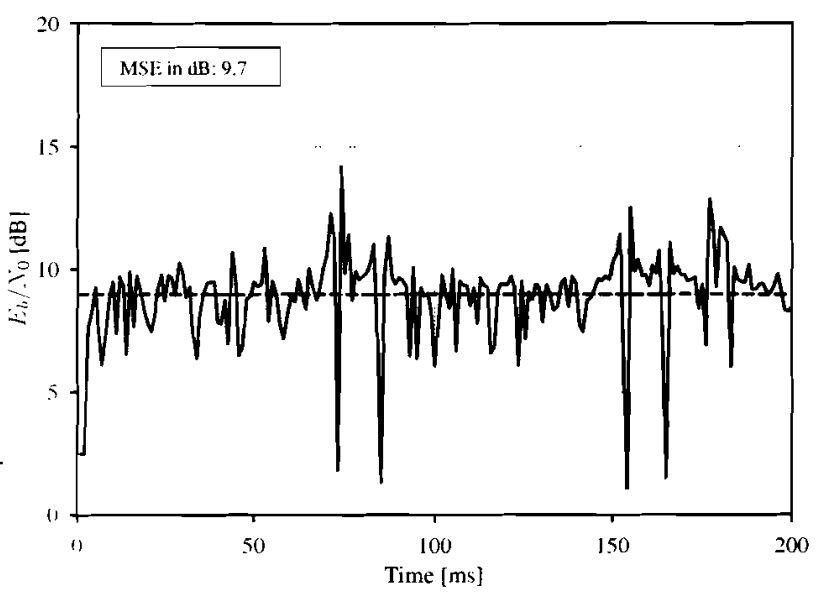

(d) Algorithm 2.

Figure 1. Sample of $E_{b} / N_{0}$ evolution for the evaluated power control algorithms, with Doppler spread $55.5 \mathrm{~Hz}$.

Table 1. MSE in dB between the actual and the target $E_{b} / N_{0}$.

\begin{tabular}{|c|c|c|c|}
\hline 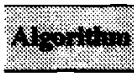 & 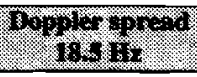 & 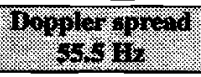 & 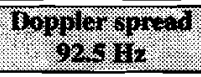 \\
\hline (1) 10 & 0.68 & 18.30 & 26.60 \\
\hline ( & -2.81 & 14.70 & 23.60 \\
\hline 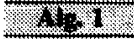 & $-7 . \overline{7} 3$ & 9.70 & 19.00 \\
\hline 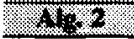 & -7.35 & $9 . \overline{70}$ & 18.30 \\
\hline
\end{tabular}

A sample of how algorithms 1 and 2 perform with respect to interference prediction is shown in fig. 2 for a single snapshot. This figure presents the behavior of the tracking performance of the interference prediction for the Doppler spread $18.5 \mathrm{~Hz}$, using eqs. (17) and (21). It can be observed that prediction of both algorithms achieves good performance for the interference. The same behavior was observed typically in all other snapshots. Evidently, as the Doppler spread increases, it occurs a performance loss caused by larger variability of the interference. Similar conclusions are valid for the path gain prediction (eqs. 16 and 20).

\subsection{SYSTEM-LEVEL RESULTS}

Now, we illustrate how the superior tracking capability of the proposed algorithms translates into a system-level advantage. In practical systems, it is difficult to keep the $E_{b} / N_{0}$ exactly at the target value, especially for high speeds [1]. Therefore, we assume an $E_{b} / N_{0}$ margin below the target $\left(E_{b} / N_{0}\right)_{t}$ in which signal quality is assumed acceptable. We simulated 5000 snapshots for several system loads and calculate the average fraction of time in which the achieved $E_{b} / N_{0}$ is below the target $\left(E_{b} / N_{0}\right)_{t}$ by margins of $0.3 \mathrm{~dB}$, $0.5 \mathrm{~dB}$ and $1 \mathrm{~dB}$. The simulated maximum load $N_{\max }$ was determined by the pole capacity equation given by [9]: 


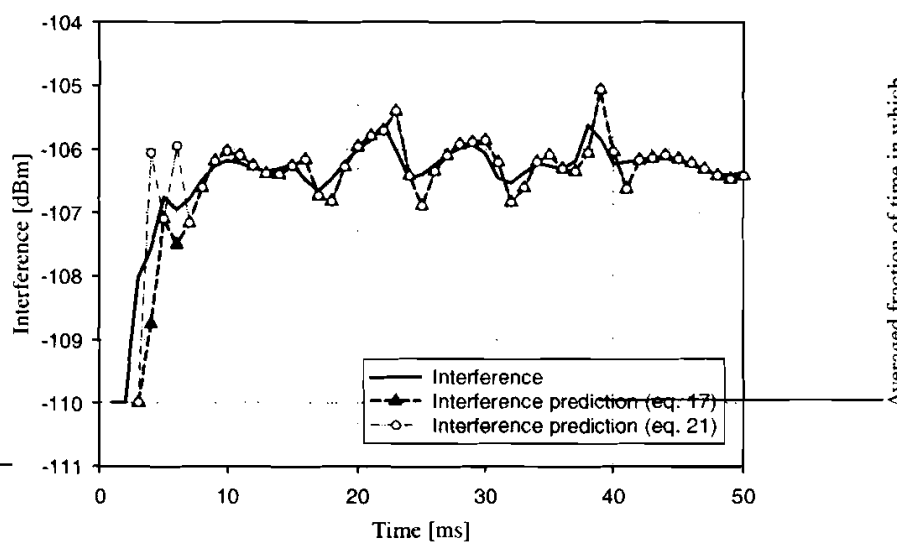

Figure 2. Comparison between actual and predicted interference using the proposed prediction methods, for the Doppler spread $18.5 \mathrm{~Hz}$.

$$
N_{\max }=1+\frac{P G}{\left(E_{b} / N_{0}\right)_{t}} \approx 17
$$

where PG is the processing gain.

Table 2 shows the average fraction of time in which the achieved $E_{b} / N_{0}$ is below the target $\left(E_{b} / N_{0}\right)_{t}$ for each simulated margin and Doppler spread $18.5 \mathrm{~Hz}$. It can be observed that the employment of the new algorithms allows for a significant capacity gain when compared to the DPC and Lee algorithm, for all simulated margins. For each margin and load system, the smallest value is highlighted, showing that the proposed algorithms outperform the DPC and Lee algorithm. Moreover, we can see that the DPC and Lee algorithm are more sensible to margin variations than the other algorithms. In other words, the proposed algorithms are more robust.

Tables 3 and 4 show the performance of the algorithms when higher Doppler spreads are considered. The Doppler spreads are $55.5 \mathrm{~Hz}$ and $92.5 \mathrm{~Hz}$. As the channel variation rate increases, a performance decrease for all algorithms is expected. However, it can be observed that the proposed algorithms still outperform the DPC algorithm and Lee algorithm.

With regard to the sensitivity of the algorithms in relation to Doppler spread, we can conclude that proposed algorithms, DPC and Lee algorithm present similar sensitivity, however the proposed algorithms are more efficient.

\subsection{EFFECT OF ACTUATION PERIOD}

In section 3 , for each proposed algorithm an assumption was done for the good working of them. This assumption consists of "the rate of power control commands is higher than the channel fading rate". In this subsection we will illustrate the limits of the actuation rate of the power control in which those assumptions are valid. Therefore, we simulate the proposed power control algorithms for some actuation rates and we compare them to DPC and Lee algorithm in 69

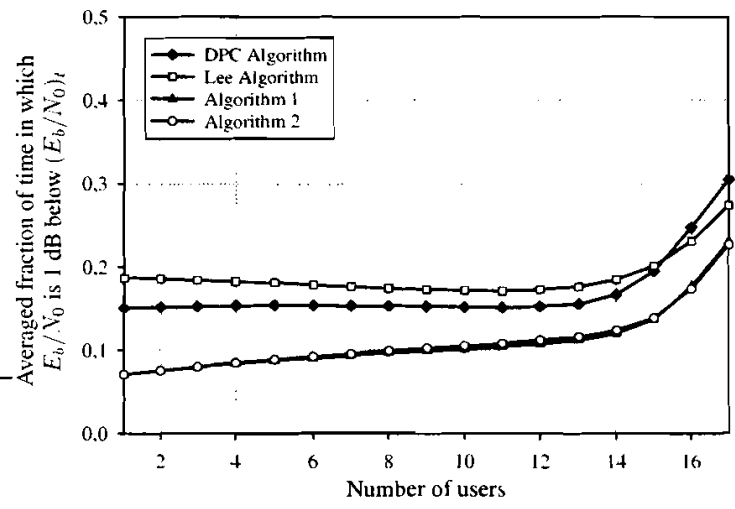

(a) Actuation period of the power control $2 \mathrm{~ms}$ and Doppler spread $18.5 \mathrm{~Hz}$.

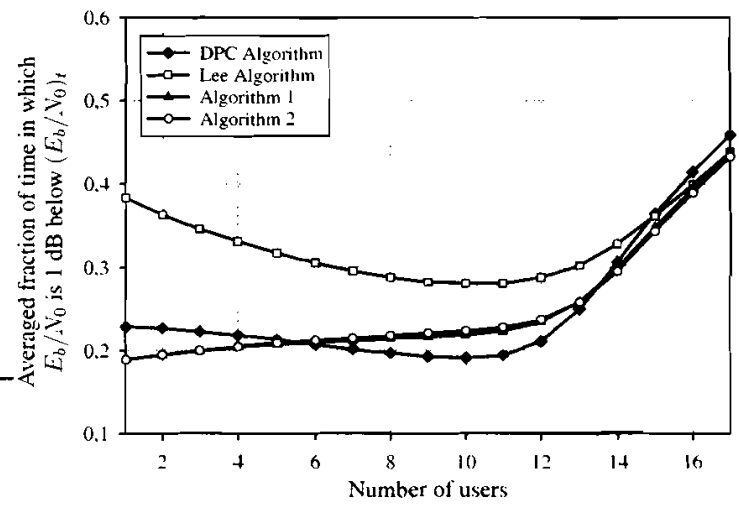

(b) Actuation period of the power control $4 \mathrm{~ms}$ and Doppler spread $18.5 \mathrm{~Hz}$

Figure 3. Averaged fraction of time in which $E_{b} / N_{0}$ is $1 \mathrm{~dB}$ below $\left(E_{b} / N_{0}\right)_{t}$ for actuation periods of the power control of $2 \mathrm{~ms}$ and $4 \mathrm{~ms}$, with Doppler spread $18.5 \mathrm{~Hz}$.

order to estimate the superior limit for the actuation-period in which the proposed algorithms outperform the DPC and Lee algorithm.

In fig. 3(a), it can be observed that the proposed algorithms make the $E_{b} / N_{0}$ to converge for the $\left(E_{b} / N_{0}\right)_{t}$ better than DPC and Lee algorithm, for an actuation period of $2 \mathrm{~ms}$. In this case, the protection margin is $1 \mathrm{~dB}$ and the Doppler spread is $18.5 \mathrm{~Hz}$. In fig. 3(b), for an actuation period of $4 \mathrm{~ms}$, DPC begins to outperform the new algorithms for some system loads. Therefore, we can infer that the superior limit for the actuation period of the proposed algorithms is between 2 and $4 \mathrm{~ms}$, for the simulated conditions. From this on, the assumed assumptions are not valid any longer and the performance of the algorithms will decrease. When we increase the Doppler spread for $55.5 \mathrm{~Hz}$ with actuation period of $2 \mathrm{~ms}$ and $3 \mathrm{~ms}$, it is expected that the performance decreases, as it can be seen in figs. 4 (a) and 4(b). In this case the limit is then between 2 and 3 ms. For higher Doppler 
Table 2. Averaged fraction of time in which $E_{b} / N_{0}$ is below $\left(E_{b} / N_{0}\right)_{t}$ for several margins, with Doppler spread $18.5 \mathrm{~Hz}$. For each margin and load system, the smallest value is highlighted.

\begin{tabular}{|c|c|c|c|c|c|c|c|c|c|c|c|c|}
\hline \multirow{2}{*}{ 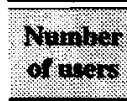 } & \multicolumn{4}{|c|}{ 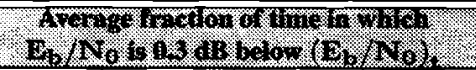 } & \multicolumn{4}{|c|}{ 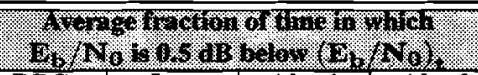 } & \multicolumn{4}{|c|}{ 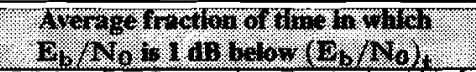 } \\
\hline & DPC & Lee & Alg. 1 & Alg. 2 & DPC & Lee & Alg. 1 & Alg. 2 & DPC & Lee & Alg. I & A $]_{\text {g. } 2}$ \\
\hline IX & 0.304 & 0.189 & 0.051 & 0.051 & 0.210 & 0.142 & 0.035 & 0.035 & 0.091 & $0 . \overline{083}$ & 0.020 & 0.020 \\
\hline W. & 0.312 & 0.195 & 0.068 & 0.073 & 0.218 & 0.146 & 0.045 & 0.048 & 0.097 & 0.086 & 0.025 & 0.026 \\
\hline 10 & 0.319 & 0.203 & 0.083 & 0.092 & 0.228 & 0.152 & 0.055 & 0.059 & 0.104 & 0.090 & 0.030 & 0.031 \\
\hline (1) & 0.400 & 0.294 & 0.185 & 0.192 & 0.324 & 0.234 & 0.151 & $\overline{0.149}$ & 0.206 & 0.158 & 0.115 & 0.108 \\
\hline
\end{tabular}

Table 3. Averaged fraction of time in which $E_{b} / N_{0}$ is below $\left(E_{b} / N_{0}\right)_{t}$ for several margins, with Doppler spread $55.5 \mathrm{~Hz}$. For each margin and load system, the smallest value is highlighted.

\begin{tabular}{|c|c|c|c|c|c|c|c|c|c|c|c|c|}
\hline \multirow{2}{*}{ 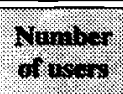 } & \multicolumn{4}{|c|}{ 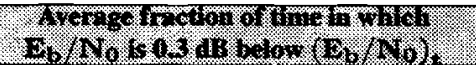 } & \multicolumn{4}{|c|}{ 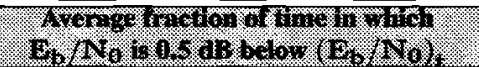 } & \multicolumn{4}{|c|}{ 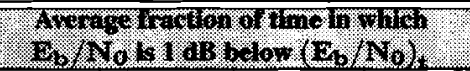 } \\
\hline & DPC & Lee & Alg. 1 & Alg. 2 & DPC & Lee & Alg. 1 & Alg, 2 & DPC & Lee & Alg. 1 & Alg. 2 \\
\hline (1) & 0.428 & 0.448 & 0.186 & 0.186 & 0.383 & 0.409 & 0.160 & 0.160 & 0.284 & 0.328 & 0.119 & 0.119 \\
\hline 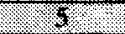 & 0.435 & 0.443 & 0.224 & 0.241 & 0.392 & 0.406 & 0.192 & 0.205 & 0.297 & 0.329 & 0.140 & 0.148 \\
\hline 10 & 0.423 & 0.436 & $0 . \overline{248}$ & 0.279 & 0.385 & $0 . \overline{402}$ & 0.214 & 0.238 & 0.299 & 0.329 & 0.158 & 0.171 \\
\hline (1) & 0.577 & $\overline{0.516}$ & 0.424 & $0.4 \overline{30}$ & $0.55]$ & 0.486 & 0.392 & 0.388 & 0.490 & 0.420 & 0.334 & 0.313 \\
\hline
\end{tabular}

Table 4. Averaged fraction of time in which $E_{b} / N_{0}$ is below $\left(E_{b} / N_{0}\right)_{t}$ for several margins, with Doppler spread $92.5 \mathrm{~Hz}$. For each margin and load system, the smallest value is highlighted.

\begin{tabular}{|c|c|c|c|c|c|c|c|c|c|c|c|c|}
\hline \multirow{2}{*}{ 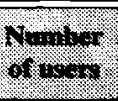 } & \multicolumn{4}{|c|}{ 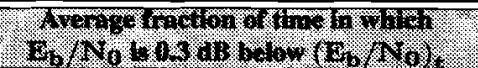 } & \multicolumn{4}{|c|}{ 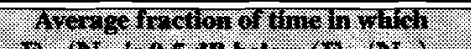 } & \multicolumn{4}{|c|}{ 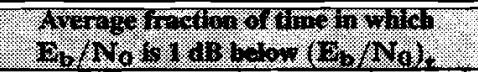 } \\
\hline & DPC & Lee & Alg. 1 & Alg. 2 & DPC & Lee & Alg. 1 & Alg. 2 & DPC & Lee & Alg. 1 & Alg. 2 \\
\hline X) & 0.4 .56 & 0.560 & 0.261 & 0.261 & 0.427 & 0.534 & $0 . \overline{244}$ & 0.244 & 0.359 & $0.47 \overline{4}$ & 0.210 & $0 . \overline{210}$ \\
\hline 3 & 0.454 & 0.537 & 0.296 & $0 . \overline{318}$ & $0.42 \overline{8}$ & 0.514 & 0.276 & 0.296 & 0.366 & 0.460 & $0 . \overline{237}$ & 0.252 \\
\hline 16 & 0.444 & 0.517 & 0.324 & 0.355 & $0 . \overline{422}$ & 0.497 & 0.305 & 0.332 & 0.368 & 0.448 & 0.264 & 0.284 \\
\hline $1 / 1$ & 0.664 & 0.628 & $0.57 t$ & 0.564 & 0.650 & 0.612 & 0.559 & 0.545 & 0.616 & 0.572 & 0.523 & 0.502 \\
\hline
\end{tabular}

spreads, the actuation period of $1 \mathrm{~ms}$ can be considered the limit as it was shown in table 4 . It is important to remember that in $3 \mathrm{G}$ systems such as WCDMA (Wideband CDMA), the actuation period of the power control is $0.67 \mathrm{~ms}$ [1], that is, below the value of $1 \mathrm{~ms}$ used in the simulation results of this paper.

\section{CONCLUSIONS AND PERSPECTIVES}

This work has presented new algorithms for power control in mobile communication systems. The proposed algorithms work well in fast time-variant channels, since they track both fast fading and interference variations. We deducted analytically their equations and observed through simulations that they are superior to the conventional DPC and Lee algorithm, for actuation period of the power control of $1 \mathrm{~ms}$, thus resulting in potential capacity gains in mobile cellular systems. As future work we will investigate theoretical aspects about the convergency of the proposed algorithms in slow fading environments. Furthermore, a more complete system-level study, including dynamic simulations in a multi-cellular network, should be accomplished.

\section{ACKNOWLEDGEMENTS}

The authors would like to express their thanks to Ericsson
Research-Brazilian Branch for the financial support.

\section{REFERENCES}

[1] A. Toskala and H. Holma, "WCDMA for UMTS - Radio Access for Third Generation Mobile Communications". Wiley, England, 2001.

[2] G. J. Lee and S. -C. Park, "Distributed Power Control in Fading Channel”, IEE, Elec. Letters, vol. 38, pp. 653-654, June 2002.

[3] G. J. Foschini and Z. Miljanic, "A Simple Distributed Autonomous Power Control Algorithm and its Convergence", IEEE Trans. Vel. Technol., vol. 42, pp. 641-646, November 1993.

[4] B. Visweswaran and T. Kiran, "Channel Prediction Based Power Control in W-CDMA Systems", 3G Mobile Communication Technologies, Conference Publication $n^{\circ} 471$, pp. 41-45, 2000.

[5] B. G. Evans, H. S. H. Gombachica and R. Tafazolli, "Predictive Power Control for S-UMTS Based on Least-Mean-Square Algorithm", $3 G$ Mobile Communication Technologies, Conference Publication $n^{\circ} 489$, pp. 128-132, 2002.

[6] F. C. M. Lau and W. M. Tam, "Novel Predictive Power Control in a CDMA Mobile Radio System", Vehicular Technology Conference, vol. 3, pp. 1950-1954, May 2000.

[7] T. M. Apostol, "Calculus", Editorial Reverté, $2^{\text {nd }}$ edition, vol. $1,1967$.

[8] W. C. Jakes, “Microwave mobile communications", Wiley, New York, $2^{\text {nd }}$ edition, 1974.

[9] J. S. Lee and L. E. Miller, "CDMA Systems Engineering Handbook", Artech House Publishers, 1997. 


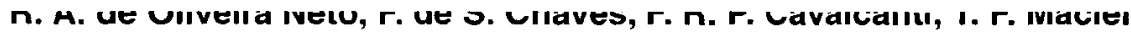
New Distributed Power Control Algorithms for Mobile Communications

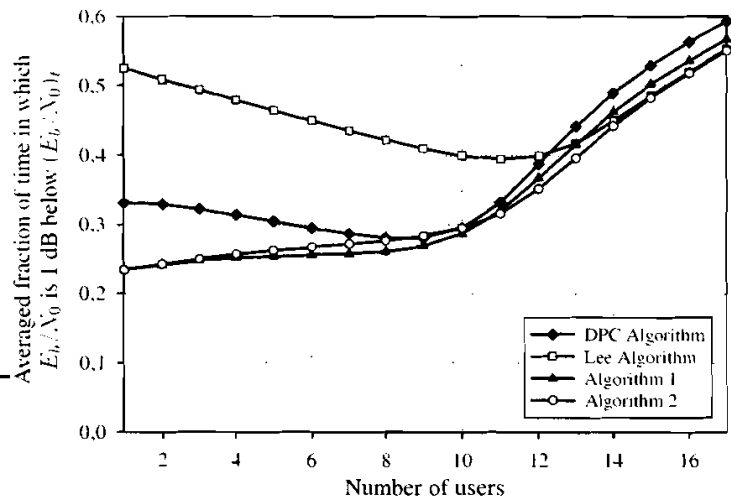

(a) Actuation period of the power control $2 \mathrm{~ms}$ and Doppler spread $55.5 \mathrm{~Hz}$

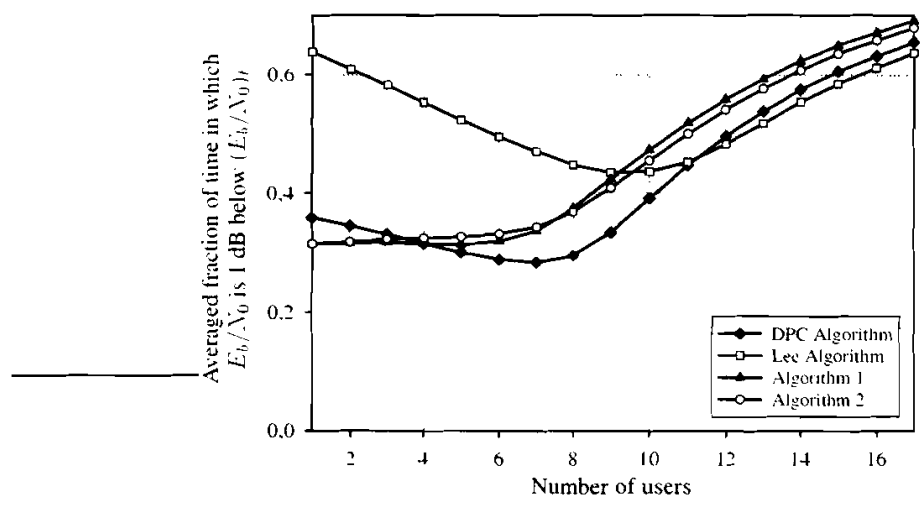

(b) Actuation period of the power control $3 \mathrm{~ms}$ and Doppler spread $55.5 \mathrm{~Hz}$

Figure 4. Averaged fraction of time in which $E_{b} / N_{0}$ is $1 \mathrm{~dB}$ below $\left(E_{b} / N_{0}\right)_{t}$ for actuation periods of the power control of $2 \mathrm{~ms}$ and $3 \mathrm{~ms}$. with Doppler spread $55.5 \mathrm{~Hz}$.

Raimundo Abreu de Oliveira Neto was born in Fortaleza, Ceará Brazil in 1977. He received his Bachelor and Master Degree in Electrical Engineering from Federal University of Ceará (UFC), Brazil, at 2001 and 2004, respectively. Since 2002 he has been with the Wireless Telecommunications Research Group (GTEL) located at the Teleinformatics Engineering Department (DETI), UFC. Presently, he is a researcher of the same research group. $\mathrm{He}$ is also member of the Brazilian Telecommunications Society (SBrt). His research interests are radio resource management and multi-access networks.

Fabiano de Sousa Chaves was born in Fortaleza, Ceará, Brazil in 1978. He received his Bachelor and Master Degrees in Electrical Engineering from Federal University of Ceará (UFC), Brazil. in 2003 and 2005 , respectively. Since 2002 he has been with the Wireless Telecommunications Research Group (GTEL) located at the Teleinformatics Engineering Department (DETI), UFC. He is also member of the IEEE and of the Brazilian Telecommunications Society (SBrt). His research interests are multiobjective optimization. noncooperative game theory, interplays between signal processing and control methodologies and radio resource management for cellular systems.

Francisco Rodrigo Porto Cavalcanti received his doctor's degree from the Communications Department of Faculty of Electrical Engineering at the University of Campinas - Unicamp, in 1999. From 1999 to 2001 he was a post-doctorate researcher at the Electrical Engineering Department of the Federal University of Ceara (UFC). Since 2002 he is an Associate Professor of the Teleinformatics Engineering Department in the same University. Currently Dr. Cavalcanti coordinates GTEL, the Wireless Telecommunications Research Group at UFC. His research interest are related to the physical layer and resource managemente aspects of wireless and mobile communication systems.

Tarcisio Ferreira Maciel was born in Fortaleza. Ceará, Brazil in 1977. He received his Bachelor and Master Degrees in Electrical Engineering from Federal University of Ceará (UFC), Brazil, in 2002 and 2004, respectively. Since 2001, he has been with the Wireless Telecommunications Research Group (GTEL) located at the Teleinformatics Engineering Department (DETI), UFC, where he developed his master studies and has been working as an associated researcher. Currently, he is developing his Ph.D. studies at the Telecommunications Institute (INT) of the Technological University of Darmstadt (TUD), in Germany. His research interests are radio resource management, smart antennas. transmil power control, and simulation of communication systems. 